\title{
3D printing of materials with spatially non-linearly varying properties
}

\section{DOI:}

10.1016/j.matdes.2018.07.012

\section{Document Version}

Accepted author manuscript

Link to publication record in Manchester Research Explorer

\section{Citation for published version (APA):}

Ren, L., Song, Z., Liu, H., Han, Q., Zhao, C., Derby, B., Liu, Q., \& Ren, L. (2018). 3D printing of materials with spatially non-linearly varying properties. Materials \& Design, 156, 470-479.

https://doi.org/10.1016/j.matdes.2018.07.012

\section{Published in:}

Materials \& Design

\section{Citing this paper}

Please note that where the full-text provided on Manchester Research Explorer is the Author Accepted Manuscript or Proof version this may differ from the final Published version. If citing, it is advised that you check and use the publisher's definitive version.

\section{General rights}

Copyright and moral rights for the publications made accessible in the Research Explorer are retained by the authors and/or other copyright owners and it is a condition of accessing publications that users recognise and abide by the legal requirements associated with these rights.

\section{Takedown policy}

If you believe that this document breaches copyright please refer to the University of Manchester's Takedown Procedures [http://man.ac.uk/04Y6Bo] or contact uml.scholarlycommunications@manchester.ac.uk providing relevant details, so we can investigate your claim.

\section{OPEN ACCESS}




\title{
3D Printing of Materials with Spatially Non-linearly Varying Properties
}

\author{
Luquan Ren ${ }^{\mathrm{a}}$, Zhengyi Song ${ }^{\mathrm{a}}$, Huili Liu ${ }^{\mathrm{a}}$, Qinghe Hanº ${ }^{\mathrm{b}}$ Brian Derbyc, \\ Qingping Liu $^{\mathrm{a} *}$, and Lei Ren ${ }^{\mathrm{d} *}$
}

${ }^{a}$ Key Laboratory of Bionic Engineering (Ministry of Education), Jilin University, Changchun, 130022, China

b Department of Radiology, The Second Hospital of Jilin University, Changchun, 130022, China

c School of Materials, University of Manchester, Manchester, M13 9PL, UK

d School of Mechanical, Aerospace and Civil Engineering, University of Manchester, Manchester, M13 9PL, UK

*Corresponding authors: Qingping Liu, E-mail: liuqp@jlu.edu.cn

Lei Ren, E-mail: lei.ren@manchester.ac.uk 


\begin{abstract}
Functionally graded materials (FGMs) with spatially varying material properties in 3D space are highly desired for a wide range of applications. Although FGMs are ubiquitous in biological materials, the fabrication of FGMs with continuous non-linear spatial variation of material properties remains a great challenge. Here we present a self-developed extrusionbased additive manufacturing system capable of fabricating FGMs with sophisticated material property distributions. The workflow involves property function modeling, gray-scale representation and control codes generation, digital material feeding and active multi-material mixing. The effectiveness of the technology is demonstrated by the successful printing of a diverse range of objects with complex spatial variations in color and $\mathrm{Al}_{2} \mathrm{O}_{3}$ concentration. In addition, by controlling the dynamic change of the different components during reactive 3D printing process, we can fabricate polyurethane (PU) objects with various gradient patterns in material mechanical properties. The results of the cantilever bending test and simulation show that the material gradient can effectively relieve the stress concentration. The proposed gradient 3D printing system opens a new avenue for manufacturing FGMs with exquisite material property distributions thus far only accessible by biological materials grown in nature.
\end{abstract}

Keywords: continuous gradient, spatial gradient, non-linear gradient, variable properties, extrusion-based 3D printing 


\section{Introduction}

Functionally graded materials (FGMs) are composite materials whose compositions, or microstructures vary progressively in one or more dimensions, resulting in a gradual change of material properties as a function of position [1]. FGMs are commonly seen in biological materials like squid beaks [2], arthropod exoskeleton [3], mussel byssus threads [4], spider fangs [5], cancellous bones [6], where material and structural efficiency has been evolutionarily optimized based on local adaption of the microstructure to demanding environment [7]. This has inspired technology for the design and fabrication of FGMs, which have great potential for biomedical, thermal, structural, optical and electronic applications [812]. However, most of the conventional techniques developed for fabricating FGMs, such as thermal spray, centrifugal casting, squeeze casting, electrophoretic deposition, and freeze drying, are only capable of constructing FGM objects of simple shapes with simple gradient patterns [13]. To produce FGM objects with complex geometries and three-dimensional (3D) compositional gradients, advanced manufacturing techniques are required.

Additive manufacturing (AM), also known as three-dimensional (3D) printing, offers a very effective platform for generating complex 3D objects from digital models $[14,15]$. In terms of FGMs, this technique benefits from its flexibility in fabrication as well as the possibilities in specifying spatial distribution of material compositions [16, 17]. Material extrusion AM technique has been demonstrated to be one of the most promising 3D printing techniques for fabricating FGMs [18]. Recently, extrusion-based technique has received attention for the fabrication of composites such as fibre reinforced composite[19-22], and has been suggested to be compatible with a wide range of materials and process conditions [21-26, 37].

In early studies, direct ink writing printing, an extrusion-based method, was developed to fabricate flat ceramic FGMs with linearly gradient of $\mathrm{Al}_{2} \mathrm{O}_{3}$ concentration along a certain direction [27, 28]. Oxman and co-workers developed a material extrusion device capable of 
mixing and fabricating silicone/polyurethane sheets with one-dimensional (1D) linear gradient hardness [30] and hydrogel samples with linearly varying chitosan/sodium alginate concentration [29]. Ober et al. designed active mixing print heads for multi-material printing of polymer structures whose local color, chemical reactivity and electrical resistivity can be tailored [31]. In addition, 3D printing of materials with gradient mechanical properties is a research hotspot of material extrusion technique, which can effectively reduce stress concentration [32, 41]. Recently, this technique also enables the fabrication of novel functionally graded 3D plasmonic constructs [33]. Overall, these attempts have appreciably pushed the limits of capability of fabricating FGMs, even though all these studies were limited to creating linear and unidimensional gradients with low precision. The state-of-theart commercial 3D printers enable the fabrication of multi-material composite with complex spatial distribution of material properties [34, 35]. However, this technique is only applicable to photosensitive resins. The privatization of materials greatly limits their further application. Therefore, the fabrication of FGMs objects with spatial and non-linear variation of material properties remains a challenge.

In this paper, we present a material extrusion AM method that enables programming and fabrication of 3D complicated FGMs for a wide range of materials. This is achieved by integrating function modeling of material properties, gray-scale representation, control codes generation, and extrusion-based 3D printing with digital materials feeding. Here, we first introduce the extrusion-based AM system, which is followed by a description of the construction of gradient function model and the generation of control codes with gray-scale. The effectiveness of the method is demonstrated by printing sophisticated FGMs objects with varying colors in $1 \mathrm{D}, 2 \mathrm{D}$ and $3 \mathrm{D}$, and graded nano- $\mathrm{Al}_{2} \mathrm{O}_{3}$ particles concentration distribution in 3D. Finally, we harness the system to fabricate polyurethane objects with different gradient patterns in material mechanical properties by reactive 3D printing. In addition, the cantilever bending experiment and simulation are performed on the object with linear gradient modulus, 
demonstrating that the gradient materials can effectively relieve the stress concentration compared to homogeneous materials. The method presented here is also versatile and can be adapted to a wide range of materials for fabricating FGMs objects with unprecedented properties.

\section{Experimental program}

\subsection{Mechanical setup and printing control system}

The mechanical setup consists of a 3-axis motion gantry, an active mixing device and a digital material feeding device (Fig. 1a). The self-made 3-axis stage with a travel of $250 \times 250$ $\times 150 \mathrm{~mm}^{3}$ was used to provide space for printing process. The self-developed active mixer has a rotation speed ranging from 0 to 100 revolutions per second, and was used to mix materials from three separate feeding pipelines in real-time. The three feeding pipelines were constructed by using flexible Teflon tubes with a diameter of $5.5 \mathrm{~mm}$, syringes (Musashi Engineering, Japan), linear motion converters (THK, Japan), two stage gear reducers (Oriental Motor Ltd., Japan), and servo motors (MiGe, China). The printing control system is made up of a 6-axis controller and self-developed software. The 6-axis controller (Leadshine Technology Ltd., China) was used to receive data from the software and generate G-codes to control the motions of the feeding syringes and 3-axis stage. The custom software was used to model gradient model and export data to the 6-axis controller.

\subsection{Materials preparation}

The red and blue color inks contain isocyanate solution, photosensitive resin (PR), FS particles and red/blue stain. The quantity of each ingredient is chosen to ensure the smooth extrusion of the mixed inks (Table S1). Isocyanate solution, the main ingredient of $\mathrm{A}$ component in 8400 flexible rubber (Hei-Cast, Japan), is used as matrix material. Photosensitive resin (White photosensitive resin, Formlabs, U.S.A.) is adopted to make the inks photocurable. Fumed silica particles (FS, AEROSIL R202, Germany, purity > 99.8\%) 
are added as rheological modifier to adjust the viscosities of the mixed inks. The red/blue stain is added to distinguish the two inks. The inks are prepared by firstly adding the photosensitive resin into the matrix solution, followed by the addition of thickeners. After the stains are added to the inks, a mixer (FSH-2A, China) is used to homogenize the inks for 10 $\min$ at 2,000 rpm.

To prepare materials for printing objects with graded nano- $\mathrm{Al}_{2} \mathrm{O}_{3}$ concentration, Alumina nanopowder (nano- $\mathrm{Al}_{2} \mathrm{O}_{3}$, Hongwu Nanometer, China, purity $>99.99 \%$, average particle size $100 \mathrm{~nm}$ ) is dispersed into the isocyanate solution, followed by the addition of $0.5 \mathrm{wt} . \%$ of surfactant (Sodium dodecyl benzene sulfonate, Guangfu Fine Chemical Research Institute, China, purity $>90.0 \%$ ) to homodisperse nano- $\mathrm{Al}_{2} \mathrm{O}_{3}$, and the addition of photosensitive resin. Thereafter, FS particles are added to modify the rheological behavior to make the inks printable. Finally, the mixture is stirred for $10 \mathrm{~min}$ at 2,000 rpm and subsequently sonicated for 40 min in a bath sonicator to remove gas bubbles.

For the material preparation of objects with gradient mechanical properties, three different inks are formulated for synthesizing PU elastomers. The raw materials (Hei Cast, Japan) consist of three components: component A containing 4,4'-Diphenyimethane diisocyanate (MDI), component B consisting of an oligomer of polyether polyol, a catalyst, and a crosslinking agent, and component $\mathrm{C}$ serving as an addition to the mixture to adjust material hardness and modulus. FS particles (AEROSIL R202, Germany) are added to modify the viscosity. Additionally, coupling reagent KH-550 (Lvxun Electronic Materials Co., Ltd., China) is used to adjust the reaction speed. The fractions of FS particles and coupling reagent are shown in Table S3. To homogenize the inks, a mixer (FSH-2A, China) running at 2,200 rpm is employed for $10 \mathrm{~min}$.

\subsection{Description of printing}


The printing parameters used to fabricate all exemplary objects in this study are listed in Table S4, and the sample's dimensions are listed in Table S5. Acrylic plates are adopted as the substrate when printing color and nano- $\mathrm{Al}_{2} \mathrm{O}_{3}$ concentration gradient objects because they have good light transmission. In the printing process, ultraviolet (UV) light with a wavelength of $395 \mathrm{~nm}$ remains illuminated on the printing platform. To make objects better cured, extra exposure is needed. The exposure time is about five to ten minutes depending on the dimensions of the printed objects. Teflon plates are used as the substrate in printing of PU objects with gradient mechanical properties because of their good anti-adhesion property. During the printing process, the as-printed PU objects cannot be completely solidified. In order to cure thoroughly, further post-processing procedures are needed. The printed objects together with the Teflon plates were placed in a blast temperature oven at $60^{\circ} \mathrm{C}$ for $2-3$ hours to achieve complete cure.

\subsection{Characterization}

All rheological property experiments are conducted with a rotational viscometer (Brookfield DV-II+ Pro Viscometer, America) using the HB 5 type of spindle. The measured results were calculated in a logarithmic scale. A portable Spectrophotometer (CM 2600D, Konica Minolta, Japan) was employed to measure the color variation performed with D65 light source at $360 \mathrm{~nm}$ to $740 \mathrm{~nm}$ with a $10 \mathrm{~nm}$ interval and 10 degrees standard observe. The selected test positions are shown in Fig. S2a and more than three measurements were conducted for each test position. Observing changes of reflectance spectra at different positions around the peaks of about $450 \mathrm{~nm}$ (blue) and $660 \mathrm{~nm}$ (red) can indicate the change of color as a function of position.

The printed cube object with $3 \mathrm{D}$ varying nano- $\mathrm{Al}_{2} \mathrm{O}_{3}$ concentration is scanned using a spiral CT machine with 256 rows (Brilliance iCT 256, Philips, Netherland) performed with an interval of $0.45 \mathrm{~mm}$, and at an X-ray voltage of $120 \mathrm{kV}$ and a current of $30 \mathrm{~mA}$. The CT scan 
images are processed to reconstruct the 3D object by using Philips IntelliSpace Portal software (Philips, Netherland). Using this software, the distribution of nano- $\mathrm{Al}_{2} \mathrm{O}_{3}$ particles on either surfaces or any section could be observed. Additionally, the CT attenuation value can reflect the concentration distribution of particles contained. We selected three sections (the middle section and the two sections closer to the two sides respectively) in each of the three axis directions (X, Y, and Z), then extracted the CT values. On each section, totally 64 test zones (forming an $8 \times 8$ equidistant grid) were conducted. The size of each zone is $3 \mathrm{~mm}$ $\times 3 \mathrm{~mm}$, and the average CT value of each zone is taken as statistics.

Material hardness is measured by using a durometer of Shore A-Hardness (Aidebao Instruments Co., Ltd., China). For the printed long plate objects, totally 11 positions are tested along the direction of length. The measurement is repeated three times at each position. For the printed square block objects shown in Fig. 9b, totally 64 points (forming an $8 \times 8$ equidistant grids in the plate plane) are measured. The test is repeated three times at each position. Elastic modulus at different positions is calculated by the compression experiment. Compressive tests were carried out using a precision test system (ZQ-990A, China). Seven test samples with the size of $8 \mathrm{~mm} \times 8 \mathrm{~mm} \times 5 \mathrm{~mm}$ were cut out of the printed long plate objects, the positions of which are shown in Fig. S7a. All samples were tested at a strain rate of $1 \mathrm{~mm} / \mathrm{min}$, and tests were stopped when the strain reaches $60 \%$. An average of 3 samples for the same position in the different objects was tested. The stress-strain curves of linear and parabolic plate objects are respectively shown in Fig. S7b and c. Elastic moduli were determined by the fitting of initial linear region.

\subsection{Cantilever bending test and simulation of graded PU plate.}

The printed plates with a linear gradient modulus was considered as a cantilever beam with the flexible end fixed, and then a upward force was applied at the rigid end to enable bending. Finite element simulations were conducted to analyse the beam bending, where discrete gradients were used instead of continuous gradients. The plate was divided into seven 
segments, each with a dimension of $3.4 \mathrm{~mm} \times 2.4 \mathrm{~mm} \times 1 \mathrm{~mm}$. The modulus of each segment is sequentially set to the measured data obtained by the compression test, and the force is applied in the same direction as in the test. In addition, the simulations of a bending beam with the same dimension and also homogeneous materials were also performed, where the constant modulus was set to be the median value of the whole plate obtained from the compression test.

\section{Results and Discussions}

\subsection{Gradient 3D printing system.}

In this paper, we successfully developed a gradient 3D printing system that is capable of fabricating objects with complicated spatially varying material properties. The 3D printing system consists of two parts: mechanical part and control part. The mechanical part is composed of 3-axis motion gantry, active mixing device and digital material feeding device (Fig. 1a). The digital material feeding device is equipped with three independently addressable syringes connected to the active mixing device with flexible tubes. The active mixing device is mounted on the 3-axis gantry for dynamically mixing of different materials. The 6-axis controller regulates the XYZ motions of the gantry and also the UVW material delivery for each separate pipeline of the digital feeding device. The controller is capable of coordinating XYUVW variables simultaneously, which can accomplish the delivery and printing of multiple materials according to predefined spatial distributions (More details are described in Experimental program).

The gradient 3D printing process includes property function modeling and gray-scale representation, control codes generation, and digital feeding and gradient printing (Fig. 1b). Property function modeling refers to the use of mathematical functions to describe the gradient distribution of material properties (details are described in Text S1). In essential, material information is numerically stored in the form of data generated by functions. Gray- 
scale images are employed to represent material composition information virtually, and the variation of gray-scale values can reflect the site-specific material composition. This is followed by the generation of control G-codes from gray-scale image, which contain the material composition data. A gray-scale image corresponds to the material property distribution in one 2D slice of a 3D object. From 0 to 254, the gray-scale image values are thereafter converted to material proportion data based on specific mapping algorithms (see Text S2 for the details). Specially, the maximum gray-scale value of 255 is defined as no extrusion. In this way, the boundary of a pre-defined pattern can be determined (an example was shown in Fig. 3a). Finally, using the generated G-codes, the 6-axis controller can dynamically regulate the material feeding rate for each individual pipeline and the 3D gantry motion to implement gradient 3D printing. The above process is integrated into a custom software, which has the design flexibility and material versatility. To demonstrate the capacity of this system to print objects with spatially non-linear gradients, graded materials with varying color, nano- $\mathrm{Al}_{2} \mathrm{O}_{3}$ concentration, and mechanical properties are fabricated, and their functionalities brought by the gradient are discussed in the following sections. 


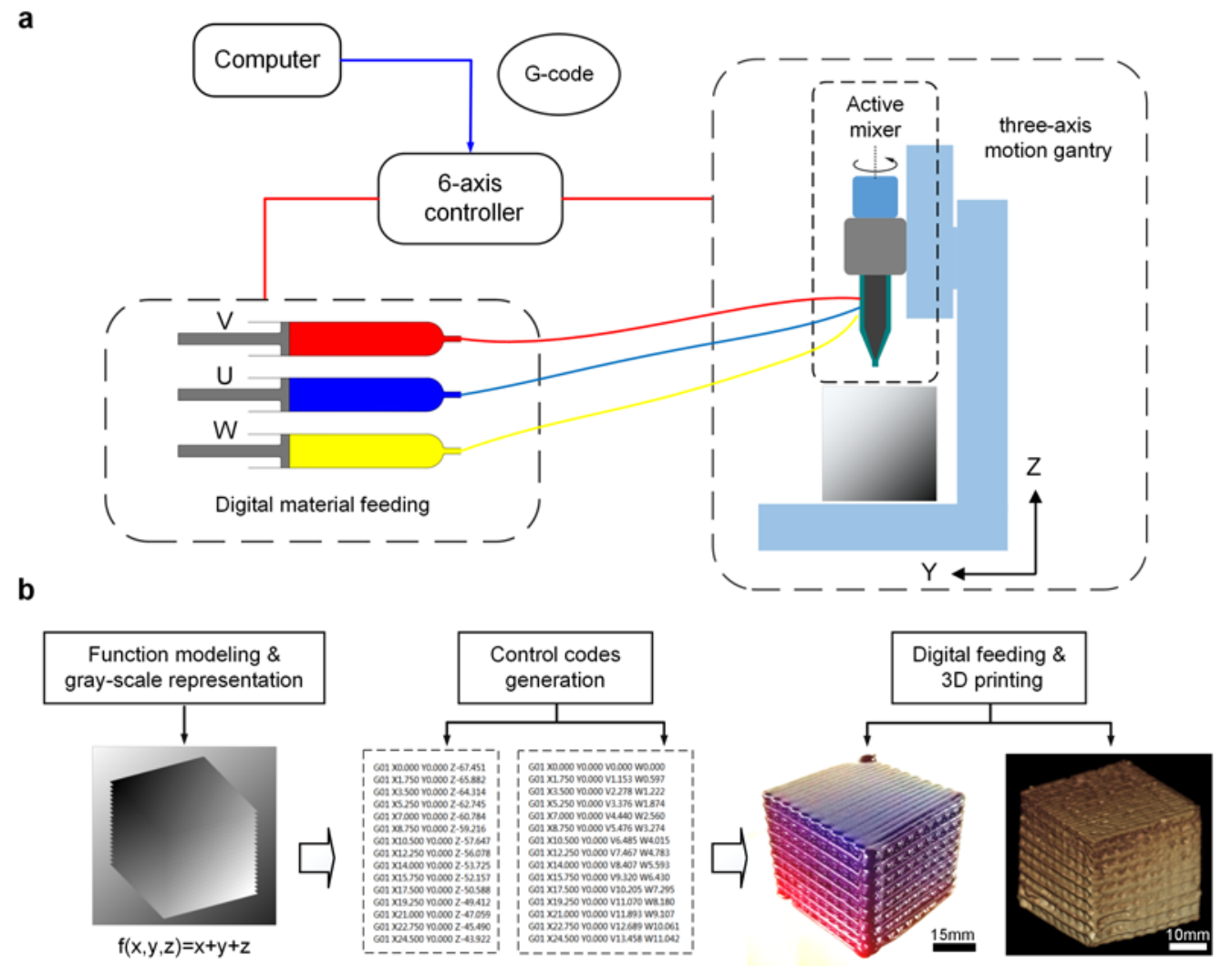

Fig. 1. Gradient 3D printing system. a) Schematics of the gradient 3D printing mechanical setup and printing control system. b) Process of gradient 3D printing.

\subsection{Printing of objects with gradient color.}

To demonstrate our ability to print gradient objects, we firstly fabricated exemplary objects with spatially varying color because it is intuitive and visible to discern the spatial variation of color. Two inks based on isocyanate solution with red and blue dyes are prepared (Fig. S1a and Table S1). The effect of fumed silica (FS) concentration on the ink rheological property (viscosity) was investigated to ensure the inks printability (Fig. S1b). In the process of printing, two inks are mixed in real-time following the G-code instructions. Essentially, the change of color reflects the variation in material composition.

We printed thin plate objects with smooth continuous 1D color gradients along their length directions (Fig. 2a-c). The color variations are expressed by the change of the relative grayscale (RG) data along the objects (Fig. 2a-c). As shown by the printed object with a linear 
color variation (Fig. 2a), the gray-scale value was converted to the mixture ratio between the two inks. The greater is the gray-scale value, more blue ink is contained in the material. The same conclusion can be derived from the variation of the corresponding reflection spectrum at different positions (Fig. S2). By modifying the linear color variation into nonlinear functions, we also fabricated objects with typical nonlinear color distributions, such as parabolic type (Fig. 2b) and sinusoidal style (Fig. 2c).
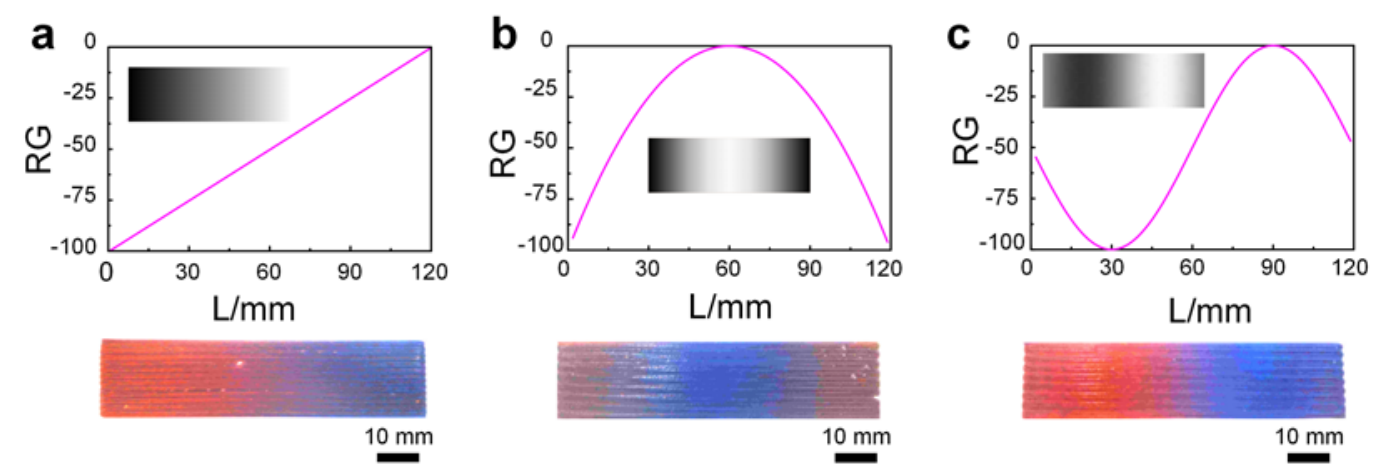

Fig. 2. Printing of objects with $1 \mathrm{D}$ gradient color. The tops are color distributions along the objects' lengths expressed by relative gray-scale data and corresponding gray-scale representations, and the bottoms are the printed 1D gradient objects: a) linear distribution, b) parabolic distribution, c) sinusoidal distribution.

a
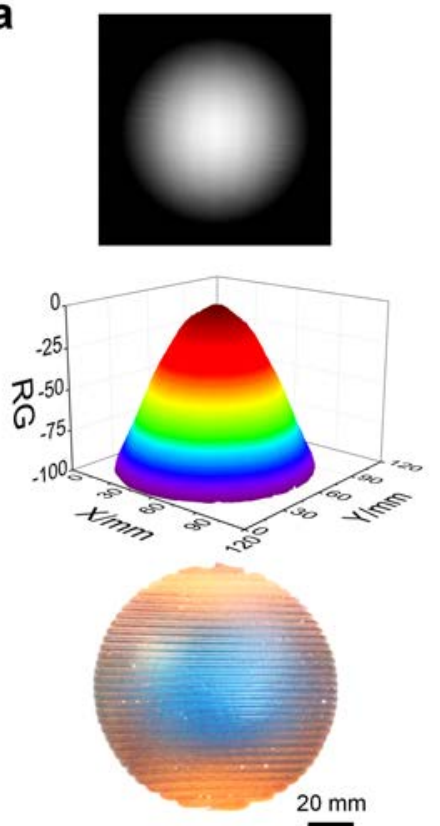

b
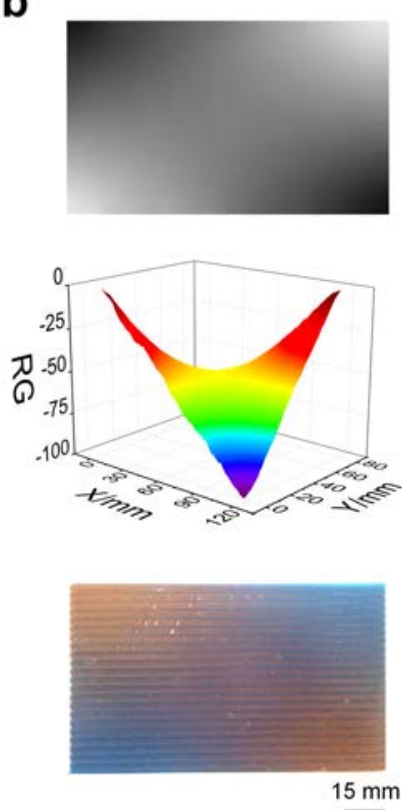

C
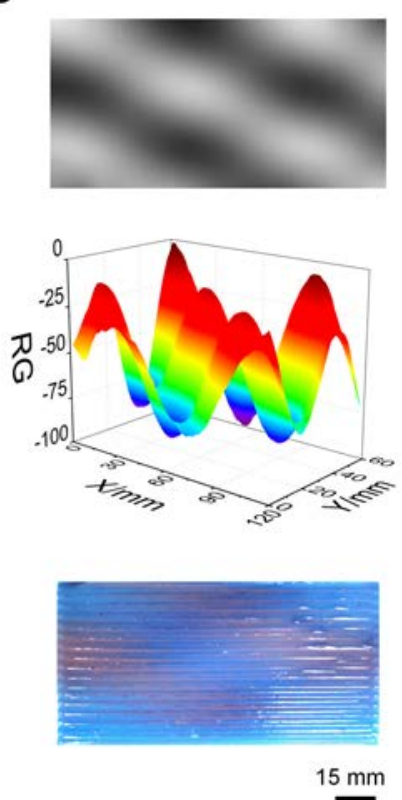

Fig. 3. Printing of objects with $2 \mathrm{D}$ gradient color. The tops are the gray-scale representations, the middles are color distributions in the plates, and the bottoms are printed 2D plates: a) radial gradient, b) saddle-like function, c) a representative complex 2D distribution. 
Moreover, we demonstrated that the system is also capable of generating objects with complex 2D material distribution (Fig. 3a-c). Similarly, the smooth continuous color distributions in the plane of the plate objects are specified by gray-scale maps. Fig. 3a shows a printed circular plate object with a radial color gradient. It is apparent that the blue content uniformly decreases from the center to the outer surface, whereas the red content uniformly increases when moving outwards from the center (Movie S1). Two rectangle plate objects with more sophisticated nonlinear color distributions in the plate planes were also fabricated (Fig. 3b and c). The generation of objects with complicated spatial property distributions depends on precise material allocation and accurate position control. Theoretically, as long as one defines a variation of material composition in the XY plane, the system could potentially produce the gradient object as pre-specified (more printed objects with complicated 2D color distributions are shown in Fig. S3).

By comparing the gray-scale images with the printed objects, we could intuitively observe the color variations along the plates or in the plate planes. This strongly demonstrates the capability of the system to print objects with $1 \mathrm{D} / 2 \mathrm{D}$ color gradients. To further illustrate the potential of this printing system in generating 3D gradients, we printed a cube with color gradient along the 3D diagonal direction (Fig. 4b and Movie S2). Although the material variations insides the object cannot be observed, the color distributions on the surfaces present color gradient patterns as pre-defined (Fig. 4a).

a

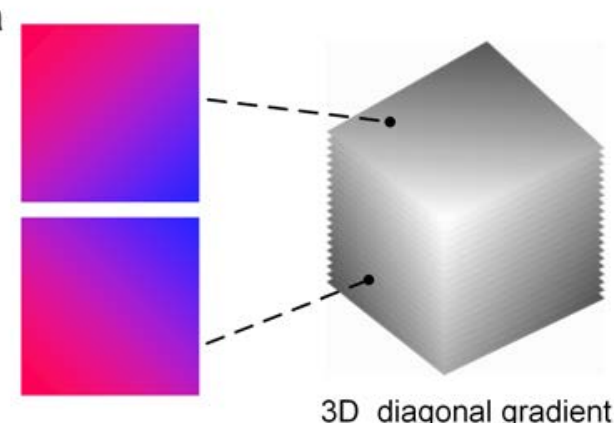

b

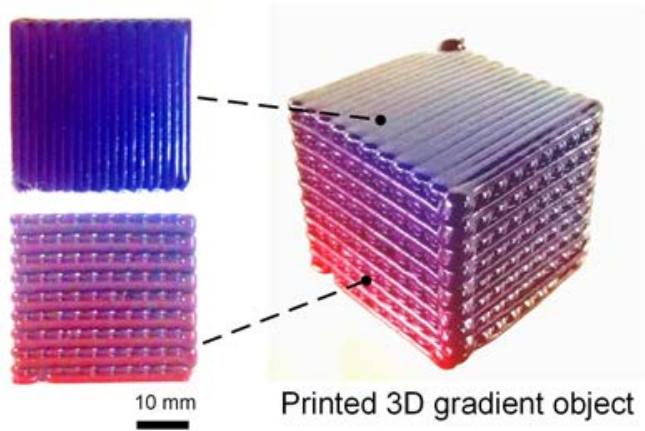


Fig. 4. Printing of objects with $3 \mathrm{D}$ gradient color along the $3 \mathrm{D}$ diagonal direction. The left is the gray-scale multi-layer representations and the color gradients represent surface material distribution. The right is the printed object and the observed top and side surfaces.

\subsection{Printing of objects with $3 \mathrm{D}$ distributed nano- $\mathrm{Al}_{2} \mathrm{O}_{3}$ concentration.}

In order to print objects with 3D distributed material contents, we prepared two inks (Table S2), with only one containing nano- $\mathrm{Al}_{2} \mathrm{O}_{3}$ particles. The nano- $\mathrm{Al}_{2} \mathrm{O}_{3}$ particle concentration is controlled by regulating the mixture volume fraction of the two inks. To ensure ink printability, moderate FS particles were added in the initial mixture solution to modify ink viscosity, and the rheological behavior was investigated (Fig. S4a). We chose nano- $\mathrm{Al}_{2} \mathrm{O}_{3}$ particle here to visually identify the particle spatial concentration via CT images. In this way, we can investigate the nano- $\mathrm{Al}_{2} \mathrm{O}_{3}$ particle distribution inside 3D objects using CT scans, where a larger CT value means more nanoparticles is contained.

A cube object was printed with $3 \mathrm{D}$ distributed nano- $\mathrm{Al}_{2} \mathrm{O}_{3}$ particle concentration along its diagonal direction (Fig. 5a). Adjacent layers vertically staggered in the printing process, the printed object kept well the pre-defined shape after curing (Fig. 5b). The printed gradient cube was analyzed and reconstructed using CT scans (Fig. 5c and Movie S3). The concentration distribution in transverse cross-sections perpendicular to all three axis directions (X, Y and Z) can be observed (Fig. 5d and Movie S4), which are in line with the pre-defined gradient pattern along 2D diagonal direction. Fig. $6 \mathrm{a}$ and $\mathrm{b}$ show the distributions of $\mathrm{CT}$ values of $\mathrm{XZ}$ cross-sections at $\mathrm{Y}$ coordinate equals to $5 \mathrm{~mm}, 17.5 \mathrm{~mm}$ and $30 \mathrm{~mm}$ respectively. It can be drawn that there is a similar distribution trend in each cross-section (Fig. 6b). In addition, there is also a linear correspondence (Fig. 6a) between the magnitude of the CT values and the position of $\mathrm{Y}$ at the same point. Similar distribution trends can also be derived from the distribution of CT values in the X and Z directions (Fig. S5 and S6). The CT scans results indicate that the nano- $\mathrm{Al}_{2} \mathrm{O}_{3}$ particles were successfully distributed in the $3 \mathrm{D}$ object as designed. 


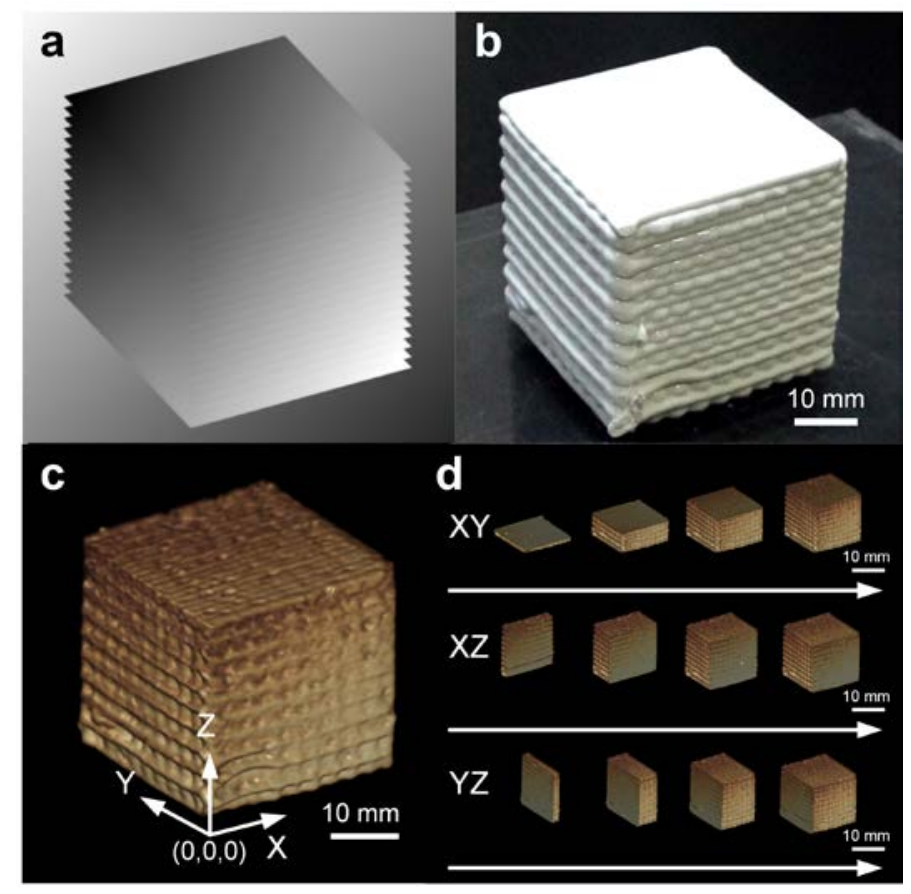

Fig. 5. Printing of objects with $3 \mathrm{D}$ graded nano- $\mathrm{Al}_{2} \mathrm{O}_{3}$ concentration. a) Multi-layer gray-scale images showing the 3D diagonal concentration distribution. b) The printed object with 3D diagonal concentration gradient. c) 3D reconstruction of the printed object using CT scans. d) Representative cross-sections perpendicular to the direction of $\mathrm{X}, \mathrm{Y}$, and $\mathrm{Z}$ axis, respectively, from the reconstructed 3D objects. The color depth on different sections indicates the concentration distribution of nano- $\mathrm{Al}_{2} \mathrm{O}_{3}$ particles.

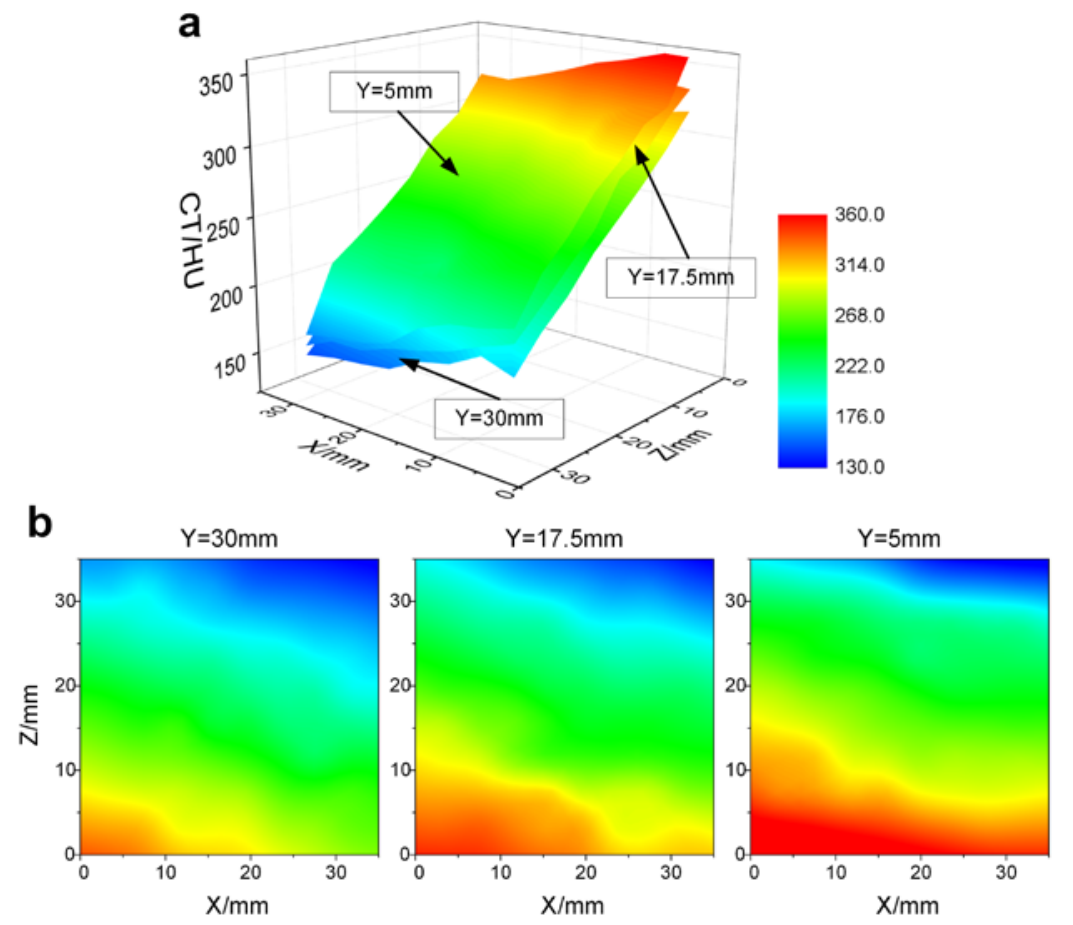

Fig. 6. CT value distributions of different sections (XZ planes), extracted from the CT reconstructed objects with printed 3D diagonal gradient. a) 3D color map surfaces of CT values. b) XYZ contours of CT values. 


\subsection{Reactive $3 D$ printing of objects with gradient mechanical properties}

To demonstrate the system's ability to print functional parts, we fabricated exemplary objects with graded material mechanical properties based on synthesis of polyurethanes (PU) by reactive 3D printing [36, 37]. Reactive 3D printing is a new 3D printing strategy, in which a material is formed by chemical reaction during deposition [38, 39]. Here, we used three different components (A, B, and C) to synthesize PU objects with gradient mechanical properties by controlling the variation of their fractions during printing process. The variation of material mechanical properties is controlled by modifying the content fraction of component $\mathrm{C}$ because it determines the number of urethane bonds - $\mathrm{NH}-\mathrm{COO}$ - formed by -OH and $-\mathrm{N}=\mathrm{C}=\mathrm{O}$ - groups in the final mixture compound (Fig. 7). In the printing process, three different components are delivered separately with delivery ratios of $1: 1$ for components A and $\mathrm{B}$. In order to ensure the normal conduct of reaction, the content fraction of component $\mathrm{C}$ is controlled not to exceed 5/7 (corresponding to the softest setting). Whereas, the hardest material is synthesized with isometric volumes of A and B without C. Notably, reaction rate control is essential to avoid nozzle clogging, and the extruded filament should be able to keep its shape. In this scenario, rheological modifiers were used to thicken the raw materials (Table S3) and the rheological properties were investigated (Fig. S4b).

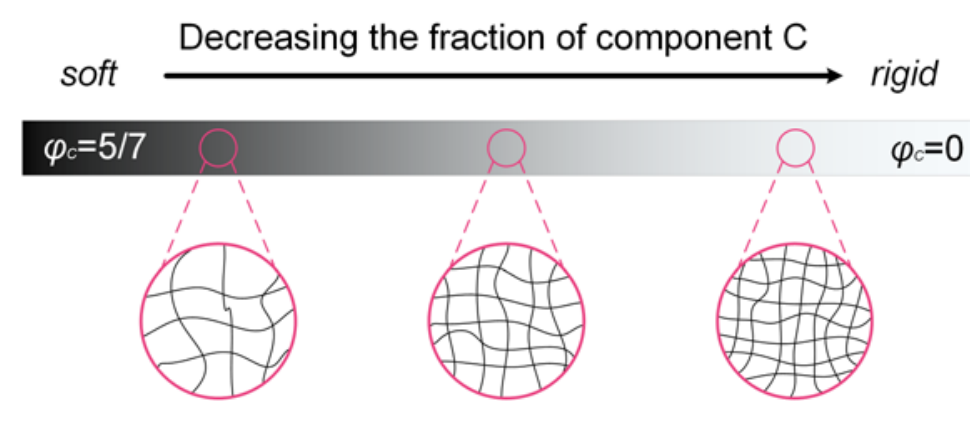

Fig. 7. Schematics of the process of modifying material mechanical properties. The density of grid line indicates the density of the urethane bonds produced by the reaction. 

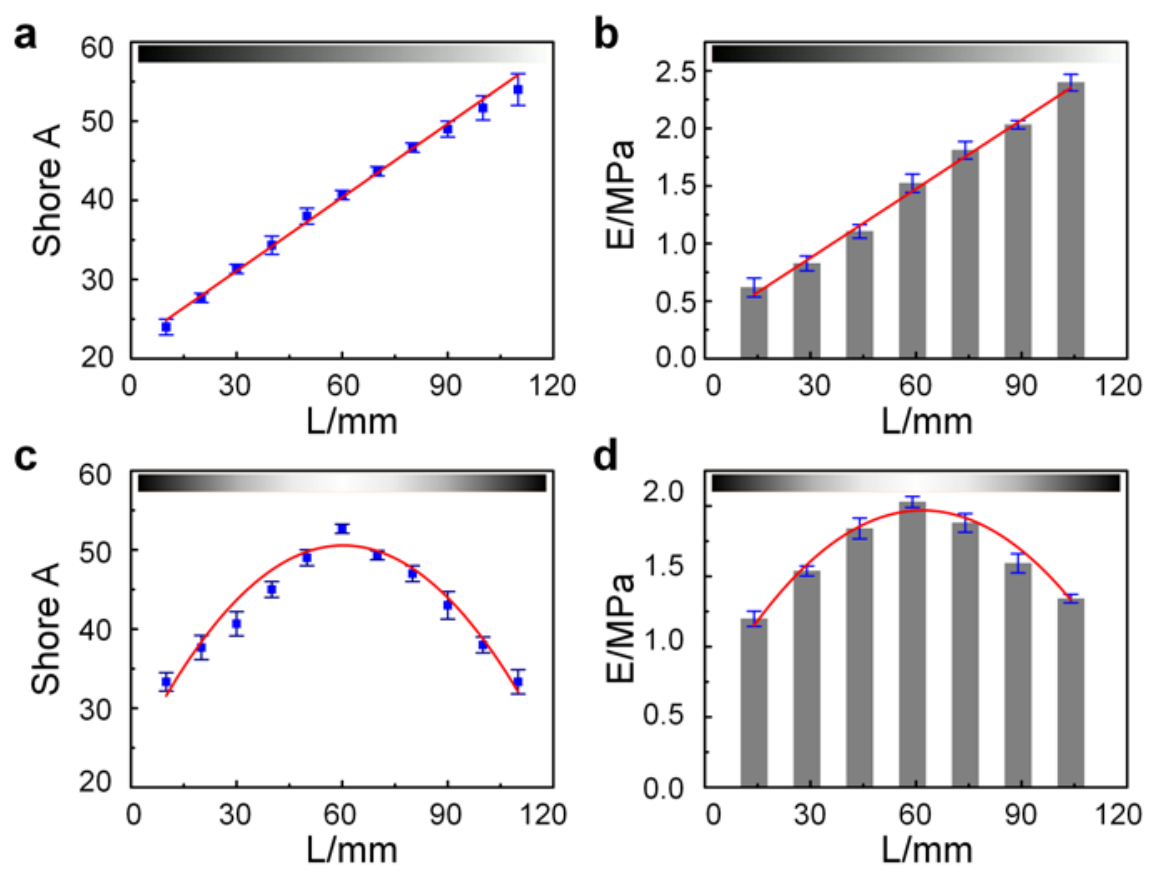

Fig. 8. Mechanical properties of printed long PU plates. The hardness a) and the modulus b) of the printed plates with linearly distributed components along their lengths as a function of position. The hardness c) and the modulus d) of the printed plates with parabolic distribution as a function of position.

We printed out a long PU plate with linearly gradient hardness (Fig. 8a and b). Along the longitudinal direction, the value of Shore A hardness increases linearly more than twice from the left end to the right end (Fig. 8a). Similar to the change in hardness, there is also a linear trend in the variation of elastic modulus (Fig. 8b). We also fabricated a long PU plate with parabolically varying hardness in the length direction (Fig. 8c and d). Both measured hardness (Fig. 8c) and elastic modulus (Fig. 8d) peak in the middle of the plate with an average trend matching the theoretical distribution function well.

Further, the distribution of mechanical properties could also be present in 2D. A square plate with a saddle-like component distribution in the object's plane was printed out (Fig. 9a,b and Movie S5). The measured hardness distribution over the plate gradually decrease as the measuring point moving towards the top right and bottom left corners from the center whilst progressively increases when moving towards the top left and bottom right corners (Fig. 9c), which is consistent with the pre-designed distribution of different components. This 
demonstrates that the proposed 3D printing technique is capable of producing functional objects with gradient material mechanical properties as designed.

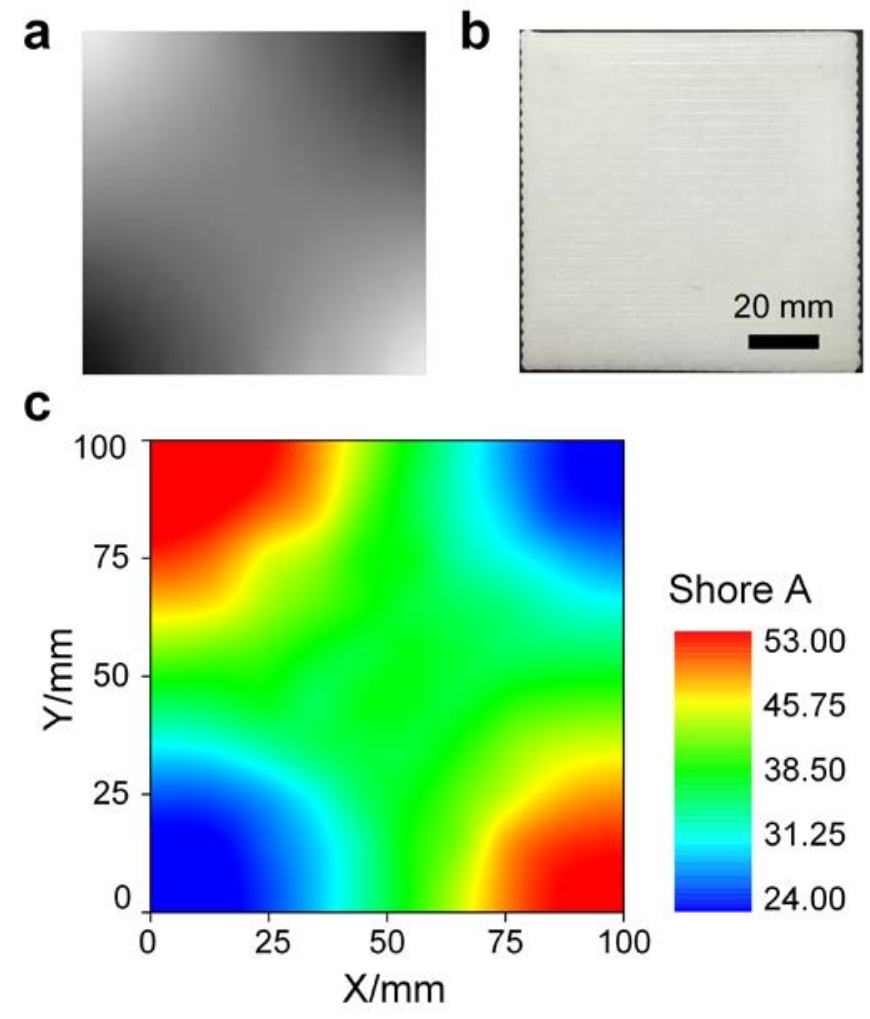

Fig. 9. a, b) Gray-scale design used for saddle-like material distribution in the object's plane and printed graded plate. c) The measured hardness distribution of the printed square plate with a saddle-like component distribution.

To better understand the effect of the gradient on the mechanical properties of the material, we performed a cantilever bending test on the printed object with linear gradient modulus (Fig. 10a). The flexible end was as fixed, which has a higher strain capacity. When bending force was applied, more bending occured on the side closer to the soft material, resulting in a significant variation in the bending degree from one side to the other. This is very similar to the finite element simulation result (see Fig. 10b). In contract, under the same loading conditions, the bending degree of the homogeneous cantilever beam does not change so much (Fig. S8). In addition, by comparing the stress distributions of homogeneous part and gradient part, the stress at the fixed end of the gradient part is smaller than that of the homogeneous part, which indicates that the material properties gradient can effectively alleviate the stress concentration. 

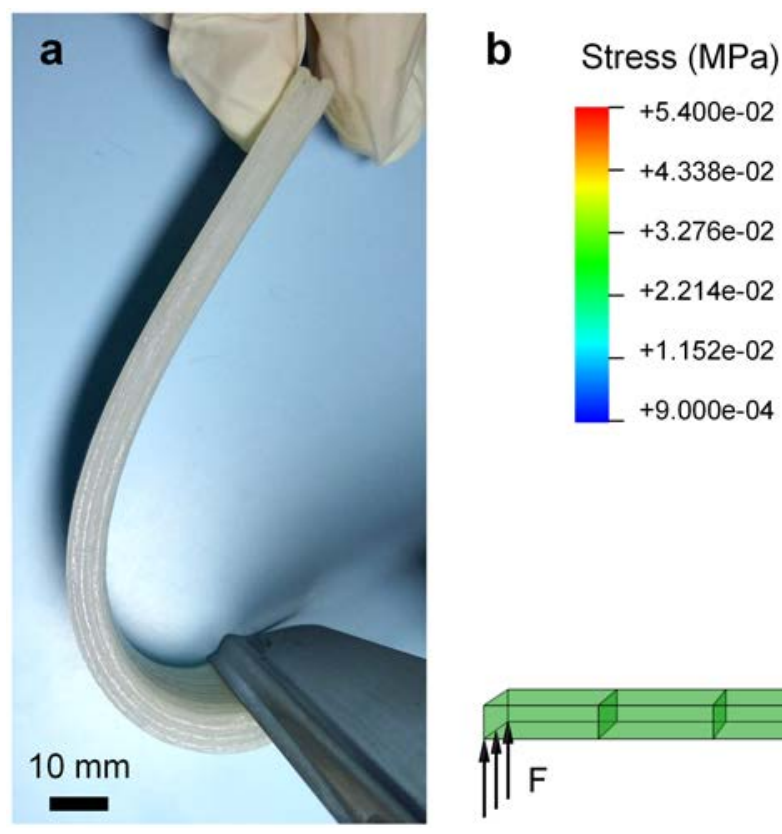

Fig. 10. Cantilever bending test of the printed object with linear gradient modulus a) and finite element simulation of the model with linear gradient modulus b).

\section{Conclusion}

In summary, we have developed a gradient 3D printing system based on material extrusion AM technique to produce FGMs objects with complicated 3D distributed properties. This technique integrates function-driven modeling, gray-scale representation and codes generation, combined with multi-material mixing, digital material feeding and extrusion-based 3D printing, forming a systematic workflow to fabricate FGMs objects. The printing of complex FGMs objects with different material properties, including color, nano- $\mathrm{Al}_{2} \mathrm{O}_{3}$ concentration, and mechanical properties, demonstrates the effectiveness and superiority of this system, which is not available using state-of-the-art technologies. Moreover, being applicable to a wide range of materials endows our system with more possibilities to create FGMs that have more superior performance in mechanical [40, 41], optical [32], thermal and electrical [42].

Due to the lack of ability to manufacture complex FGMs objects, the superior properties and great potentials of FGMs have not yet been fully explored. Our strategy presents an effective tool to autonomously design and fabricate complex 3D gradient objects. As 
demonstrated by the printed exemplary samples, the proposed gradient 3D printing system has the ability to produce heterogeneous objects with non-linearly varying material properties in the 3D space. Although printing precision and shaping complexity could be further improved, it is undoubtable that significant progress has been made in this report compared to previous works. We have succeeded in extending the gradient printing from producing simple 1D gradient to fabricating objects with complicated 2D and 3D non-linear distributions of a large variety of material properties. Future exploration and acknowledgment of FGMs will enable us to make the best use of their performances and facilitate the advances and developments of many areas, including aerospace, energy [43], defence, biomedical [44], opto-electronics, robotics etc. All together, the proposed gradient 3D printing system opens a new avenue for manufacturing FGMs with exquisite material property distributions thus far only accessible by biological materials grown in nature.

\section{Acknowledgements}

This research was supported by the National Key Research and Development Program of China (No. 2018YFB1105103) and the Key Scientific and Technological Project of Jilin Province (No. 20170204061GX). 


\section{References}

[1] M. Niino, T. Hirai, R. Watanabe, The functionally gradient materials, J. Jap. Soc. Comp. Mater. 13 (1987) 257-264.

[2] A. Miserez, T. Schneberk, C. Sun, F.W. Zok, J.H. Waite, The transition from stiff to compliant materials in squid beaks, Science 319 (2008) 1816-1819.

[3] B. Shmuel, Z. Paul, A.S. Ali, M. Admir, F. Peter, S. Amir, B. Amir, A. Barara, Enamellike apatite crown covering amorphous mineral in a crayfish mandible, Nat. Commu. 3 (2012) 839.

[4] M.J. Harrington, A. Masic, N. Holtenandersen, J.H. Waite, P. Fratzl, Iron-clad fibers: a metal-based biological strategy for hard flexible coatings, Science 328 (2010) 216-220.

[5] Y. Politi, M. Priewasser, E. Pippel, P. Zaslansky, J. Hartmann, S. Siegel, C. Li, F.G. Barth, P. Fratzl, A spider's fang: how to design an injection needle using chitin-based composite material, Adv. Funct. Mater. 22 (2012) 2519-2528.

[6] G.M. Genin, A. Kent, V. Birman, B. Wopenka, J.D. Pasteris, P.J. Marquez, S. Thomopoulos, Functional grading of mineral and collagen in the attachment of tendon to bone, Biophys. J. 97 (2009) 976-985.

[7] A.R. Studart, Biological and bioinspired composites with spatially tunable heterogeneous architectures, Adv. Funct. Mater. 23 (2013) 4423-4436.

[8] W. Pompe, H. Worch, M. Epple, W. Friess, M. Gelinsky, P. Greil, U. Hempel, D. Scharnweber, K. Schulte, Functionally graded materials for biomedical applications, Mater. Sci. Eng. A 362 (2003) 40-60.

[9] Z. Jin, R.C. Batra, Thermal shock resistance of functionally graded materials, Acta Mater. 52 (2004) 4961-4972.

[10] J.F. Bartolomé, J.S. Moya, J. Requena, J. Llorca, M. Anglada, Fatigue crack growth behavior in mullite/alumina functionally graded ceramics. J. Am. Ceram. Soc. 81 (2010) 1502-1508. 
[11] E. Müller, Č. Drašar, J. Schilz, W. Kaysser, Functionally graded materials for sensor and energy applications, Mater. Sci. Eng. A 362 (2003) 17-39.

[12] I. Bharti, N. Gupta, K.M. Gupta, Novel applications of functionally graded nano, optoelectronic and thermoelectric materials, Int. J. Mater. Mech. Manuf. 1 (2013) 221224.

[13] M. Naebe, K. Shirvanimoghaddam, Functionally graded materials: a review of fabrication and properties, Appli. Mater. Today 5 (2016) 223-245.

[14] H. Bikas, P. Stavropoulos, G. Chryssolouris, Additive manufacturing methods and modelling approaches: a critical review, Int. J. Adv. Manuf. Technol. 83 (2016) 389-405.

[15] S. Huang, P. Liu, A. Mokasdar, L. Hou, Additive manufacturing and its societal impact: a literature review. Int. J. Adv. Manuf. Technol. 67 (2013) 1191-1203.

[16] N. Oxman, Variable property rapid prototyping, Virt. Phys. Prototyp. 6 (2011) 3-31.

[17] E. Pei, G.H. Loh, D. Harrison, H.D.A. Almeida, M.D. Monzon Verona, R. Paz, A study of $4 \mathrm{D}$ printing and functionally graded additive manufacturing, Assembly Autom. 37 (2017) 147-153.

[18] G.E. Hilmas, J.L. Lombardi, R.A. Hoffman, Advances in the fabrication of functionally graded materials using extrusion freeform fabrication, in Functionally Graded Materials, 1997, pp. 319-324.

[19] B.G. Compton, J.A. Lewis, 3d-printing of lightweight cellular composites, Adv. Mater. 26 (2015) 5930-5935.

[20] G.A. Sydney, E.A. Matsumoto, R.G. Nuzzo, L. Mahadevan, J.A. Lewis, Biomimetic 4D printing, Nat. Mater. 15 (2016) 413-418.

[21] G.D. Goh, V. Dikshit, A.P. Nagalingam, G.L. Goh, S. Agarwala, S.L. Sing, J. Wei, W.Y. Yeong, Characterization of mechanical properties and fracture mode of additively manufactured carbon fiber and glass fiber reinforced thermoplastics, Mater. Des. 137 (2018) 79-87. 
[22] N. Mohan, P. Senthil, S. Vinodh, N. Jayanth, A review on composite materials and process parameters optimisation for the fused deposition modelling process, Virt. Phys. Prototyp. 12 (2017) 47-59.

[23] J. A. Lewis, Direct ink writing of 3d functional materials, Adv. Funct. Mater. 16(2006) 2193-2204.

[24] J.A. Lewis, J.E. Smay, J. Stuecker, J. Cesarano, Direct ink writing of three-dimensional ceramic structures, J. Am. Ceram. Soc. 89 (2010) 3599-3609.

[25] H.W. Kang, S.J. Lee, I.K. Ko, C. Kengla, J.J. Yoo, A. Atala, 3d bioprinting system to produce human-scale tissue constructs with structural integrity, Nat. Biotechnol. 34 (2016) 312-322.

[26] D. Kokkinis, M. Schaffner, A.R. Studart, Multimaterial magnetically assisted 3D printing of composite materials, Nat. Commun. 6 (2015) 8643.

[27] J. Wang, L.L. Shaw, Fabrication of functionally graded materials via inkjet color printing, J. Am. Ceram. Soc. 89 (2006) 3285-3289.

[28] P. Gingter, A. M. Waetjen, M. Kramer, R. Telle, Functionally graded ceramic structures by direct thermal inkjet printing, J. Ceram. Sci. Technol. 6 (2015) 119-124.

[29] L. Mogas-Soldevila, J. Duro-Royo, N. Oxman, Water-based robotic fabrication: largescale additive manufacturing of functionally graded hydrogel composites via multichamber extrusion, 3D Print. Addit. Manuf. 1 (2014) 141-151.

[30] N. Oxman, E. Tsai, M. Firstenberg, Digital anisotropy: a variable elasticity rapid prototyping platform, Virt. Phys. Prototyp. 7 (2012) 261-274.

[31] T.J. Ober, D. Foresti, J.A. Lewis, Active mixing of complex fluids at the microscale, Proc. Natl. Acad. Sci. U.S.A. 112 (2015) 12293-12298.

[32] S.E. Bakarich, R. Gorkin, R. Gately, S. Naficy, M. in het Panhuis, G.M. Spinks, 3D printing of tough hydrogel composites with spatially varying materials properties, Addit. Manuf. 14 (2017) 24-30. 
[33] A.P. Haring, A.U. Khan, G. Liu, B.N. Johnson, 3d printed functionally graded plasmonic constructs, Adv. Opt. Mater. 5 (2017) 1700367.

[34] C. Bader, D. Kolb, J.C. Weaver, N. Oxman, Data-driven material modeling with functional advection for 3D printing of materially heterogeneous objects, 3D Print. Addit. Manuf. 3 (2016) 71-79.

[35] C. Bader, N. Oxman, Recursive symmetries for geometrically complex and materially heterogeneous additive manufacturing, Comput. Aided Des. 81 (2016) 39-47.

[36] P. Kröber, J.T. Delaney, J. Perelaer, U.S. Schubert, Reactive inkjet printing of polyurethanes, J. Mater. Chem. 19 (2009) 5234-5238.

[37] M. Müller, Q.U. Huynh, E. Uhlmann, M.H. Wagner, Study of inkjet printing as additive manufacturing process for gradient polyurethane material, Prod. Eng. 8 (2014), 25-32.

[38] P.J. Kitson, S. Glatzel, W. Chen, C.G. Lin, Y.F. Song, L. Cronin, 3D printing of versatile reactionware for chemical synthesis, Nat. Prot. 11 (2016) 920-936.

[39] C. Sturgess, C.J. Tuck, I.A. Ashcroft, R.D. Wildman, 3D reactive inkjet printing of polydimethylsiloxane, J. Mater. Chem. C 5 (2017) 9733-9743.

[40] B. Wang, A.J. Benitez, F. Lossada, R. Merindol, A. Walther, Bioinspired mechanical gradients in cellulose nanofibril/polymer nanopapers, Angew. Chem. Int. Ed. 55 (2016) 5966-5970.

[41] D. Kokkinis, F. Bouville, A.R. Studart, 3D printing of materials with tunable failure via bioinspired mechanical gradients, Adv. Mater. 30 (2018) 1705808.

[42] Y. Zhuang, W. Song, G. Ning, X. Sun, Z. Sun, G. Xu, B. Zhang, Y. Chen, S. Tao, 3Dprinting of materials with anisotropic heat distribution using conductive polylactic acid composites, Mater. Des. 126 (2017) 135-140.

[43] N.W. Bartlett, M.T. Tolley, J.T.B. Overvelde, J.C. Weaver, B. Mosadegh, K. Bertoldi, G.M. Whitesides, R.J. Wood, A 3D-printed, functionally graded soft robot powered by combustion, Science 349 (2015) 161-165. 
[44] L.G. Bracaglia, B.T. Smith, E. Watson, N. Arumugasaamy, A.G. Mikos, J.P. Fisher, 3D Printing for the design and fabrication of polymer-based gradient scaffolds, Acta Biomater. 56 (2017) 3-13. 